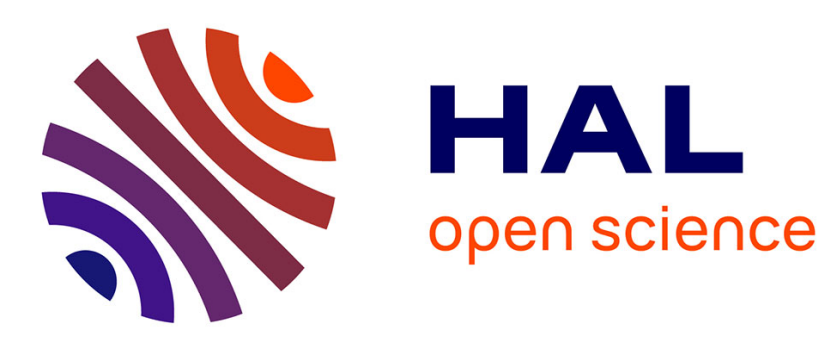

\title{
Conductivity of weak electrolytes for buffer solutions: Modeling within the mean spherical approximation
}

\author{
O. Bernard, J. Aupiais
}

\section{To cite this version:}

O. Bernard, J. Aupiais. Conductivity of weak electrolytes for buffer solutions: Modeling within the mean spherical approximation. Journal of Molecular Liquids, 2018, 272 (15 Décembre 2018), pp.631637. 10.1016/j.molliq.2018.09.103 . hal-01974195

\section{HAL Id: hal-01974195 \\ https://hal.sorbonne-universite.fr/hal-01974195}

Submitted on 8 Jan 2019

HAL is a multi-disciplinary open access archive for the deposit and dissemination of scientific research documents, whether they are published or not. The documents may come from teaching and research institutions in France or abroad, or from public or private research centers.
L'archive ouverte pluridisciplinaire HAL, est destinée au dépôt et à la diffusion de documents scientifiques de niveau recherche, publiés ou non, émanant des établissements d'enseignement et de recherche français ou étrangers, des laboratoires publics ou privés. 


\title{
Conductivity of weak electrolytes for buffer solutions: Modeling within the Mean Spherical Approximation.
}

\author{
O. Bernard ${ }^{a 1}$, J. Aupiais ${ }^{b}$ \\ a Sorbonne Université, CNRS, Physicochimie des électrolytes et nanosystèmes inter- \\ faciaux, UMR PHENIX, F-75005, Paris, France \\ ${ }^{b}$ CEA, DAM, DIF, F-91297 Arpajon Cedex, France
}

\begin{abstract}
The purpose of this study is to predict the electrical conductivity of some weak electrolytes used in buffer solutions. The acid-base reactions occurring between the different species in solution are evaluated taking into account the activity coefficients. The deviations from ideality necessary to describe the equilibrium and transport properties are estimated within the mean spherical approximation (MSA). Results from theoretical expressions of the conductivity of electrolyte mixtures are compared with experimental results from the literature in the case of acetate, carbonate and bicarbonate solutions. In order to characterize all the ions that may be present, we also studied the ability of our theory to describe some strong alkaline or acid solutions.
\end{abstract}

\section{Introduction}

Electroanalytical methods such as conductivity, zetametry or capillary electrophoresis (CE) are very useful to characterize the charge and size of particles in solution. They may also provide information on the titration and complexation of charged species studied in the presence of various ligands. In particular, capillary electrophoresis has found applications for speciation studies [1-5]. As examples, CE has shown its capability to study the inorganic speciation of $\mathrm{Al}^{3+}$ with $\mathrm{F}^{-}$and $\mathrm{C}_{2} \mathrm{O}_{4}^{2-}[6]$, of mono-, di- and trivalent cations with $\mathrm{SO}_{4}^{2-}$ [7], of $\mathrm{UO}_{2}^{2+}$ with $\mathrm{IO}_{3}^{-}$[8] by U.V. detection, and also to determine thermodynamic parameters. These solutions can, by their origin, be naturally concentrated [9-11]. On the other hand, in order to improve the resolution of electrophoresis peaks, the measurements are often performed in the presence of a concentrated supporting electrolyte solution. The position of the electrophoresis peaks can be strongly affected by the presence of this supporting electrolyte. Furthermore, the stability or conformational changes of certain molecules such as proteins are strongly dependent on the $\mathrm{pH}$ of the solution. Buffer solutions are often used for this purpose.

Theoretical models currently used to describe electrophoresis (in this field) (DebyeHückel or extended Debye-Onsager-Bjerrum models) are limited to the $0.01 \mathrm{M}$ to $0.1 \mathrm{M}$ range, by the non idealities in transport, despite the fact that empirical models for the non ideality in thermodynamics (activity coefficients) can be used [12-15]. However some

\footnotetext{
${ }^{1}$ to whom any correspondence should be addressed, E-mail: olivier.bernard@upmc.fr
} 
progress in the transport theories, have been made in the last decades. They have been successfully applied to the description of various quantities such as self-diffusion of ions [16-20], mutual diffusion coefficients of electrolytes $[21,22]$ or the electrical conductivity of ionic solutions [23-31]. These models allow one to describe quantitatively the evolution of the transport coefficients for concentrations of the range of 1 to $2 \mathrm{M}$ for electrolyte mixtures containing small ions [32-35]. The description of the electrophoretic mobility of charged tracers in solutions of binary electrolyte has been also carried out [36]. However, in these works, the solutions studied contained only simple salts such as alkali or alkaline earth halides.

On the other hand, the characterization of charged molecules in solution requires taking into account the formation of different chemical complexes of the molecules with ions or between the various ions in solution. In particular when the charge and the conformation of the molecules is $\mathrm{pH}$-dependent, a buffer solution is generally used in the supporting electrolyte.

Other experimental measurements such as zetametry and conductimetric titration may require the use of solutions of rather high ionic strength. Thus, in order to better separate the effects due to various acid-base sites present on a molecule, it may be useful to increase the concentration of supporting electrolyte to screen the interactions between neighboring sites on the molecules.

Moreover, the analysis of molecules of biological origin requires keeping their solution at a controlled $\mathrm{pH}$ in order to not modify their structure or their properties. In the case of proteins, it may also be necessary to maintain the ionic composition of the natural solution in order to be as close as possible to real conditions. These solutions do not contain exclusively alkali or alkaline earth halides but also salts of weak acids.

It would therefore be desirable to better characterize the transport properties of ions involved in acid-base reactions. Obviously, the study of the conductivity of these ions in solution gives us information about their migration under the action of an electric field. For a long time many experimental studies of the conductivity of weak acids or of salts of these acids have been carried out in dilute solutions [37-44]. Nowadays, the conductivity of dilute aqueous solutions of this type of salt still gives rise to many experimental studies [45-49]. These solutions often contain a mixture of more than two types of ions. Expressions developed by Onsager, Fuoss and Pitts [50-52] for binary electrolytes are not applicable here. When these solutions are diluted enough, their conductivity can be described using the expressions established for mixtures with any number of ionic species [53-55].

On the other hand, the understanding of the conductivity of these electrolytes in concentrated solution is much less well known. The expressions used previously to describe the conductivity of concentrated electrolyte mixtures [34,35] must be able to account for the variations observed for these salts of weak acids. We therefore propose to apply these expressions to this type of electrolyte in concentrated solution.

In addition, when the concentration of the supporting electrolyte is high, deviations from ideality (activity coefficients) must be taken into account in order to quantitatively describe chemical equilibria between the complexes formed and the dissociated ions. For high concentrations, progress has been made in the description of deviations from ideal- 
ity since the time of Debye or Pitzer. Applications of the mean spherical approximation (MSA) theory $[56,57]$ have allowed to describe osmotic and activity coefficients of real electrolyte solutions for concentrations of the order of 1 or $2 \mathrm{M}$ [58-60]. It is then possible to consider the modelling of ionic mobilities in solutions with both transport and equilibrium non idealities, as well as speciation phenomena (complex formation).

In this work, in a first approach we have chosen to describe sodium acetate solutions. It is a simple salt, containing a rather small anion and coming from a monoacid. It is relatively common and appears in the composition of buffer solutions. In addition, experimental conductivity data at high concentrations are available for this salt. Thereafter, we were interested in carbonate and bicarbonate solutions. The characterization of transport properties of charged species in aqueous solutions containing carbonate and bicarbonate ions gave rise to several studies previously $[11,61,62]$. More generally, the salts such as $\mathrm{Na}_{2} \mathrm{CO}_{3}$ and $\mathrm{NaHCO}_{3}$ are used also to make basic buffers. The properties of carbonate and bicarbonate ions are important to take into account the dissolution of $\mathrm{CO}_{2}$ in aqueous solutions $[63,64]$. Description of the transport properties of carbonate and bicarbonate solutions is also important to analyse electrical conductance measurements used in the assessment of natural water quality [65-68]. Then we have chosen to describe the conductivity in solutions containing $\mathrm{KHCO}_{3}$ and $\mathrm{Na}_{2} \mathrm{CO}_{3}$. All the solutions studied so far are alkaline. Sometimes in order to limit the hydrolysis of charged species studied, the latter can be dissolved in an acid solution. It is interesting to characterize these solutions as well. Studies of the conductivity of weak acid solutions, such as acetic acid, have been conducted for a long time. Thus, in view of its dissociation constant, the decay of the conductivity of acetic acid takes place essentially for concentrations below 0.2 M. Many studies already describe this acid and the salts obtained by its neutralization $[37,46,49,69,70]$. In this article we wanted to evaluate the ability of our transport model to describe concentrated acidic solutions. In order to overcome the speciation problems specific to weak acids we are interested in an acid which is considered to be completely dissociated, namely $\mathrm{HCl}$ in water.

The chemical equilibria linking the different species in solution are evaluated taking into account the activity coefficients. The deviations from ideality necessary to describe the equilibrium and transport properties are determined using the MSA theory. In the next section we present the theoretical model used to describe both the activity coefficients and the conductivity in an arbitrary mixture of electrolytes. In the following section, this theoretical model is applied to the description of the different binary salts and mixtures thereof which make up the supporting electrolyte selected.

\section{Theory}

\subsection{Description of the speciation}

In our model the solvent is seen as a continuum only characterized by its dielectric constant and viscosity. The ions $i$ are represented as charged hard spheres, of charge $z_{i} e$ and diameter $\sigma_{i}$ (with $z_{i}$ the valence of the ion $i$ and $e$ the charge of the proton). In order to 
calculate the various deviations from ideality occurring in the equilibrium and transport processes in solution, the distribution functions between the ions are deduced from the mean spherical approximation. The salts studied can be seen as the product of the reaction of a weak acid with a strong base, $\mathrm{NaOH}$ or $\mathrm{KOH}$ depending on the nature of the cation of the salt $\left(\mathrm{Na}^{+}\right.$or $\left.\mathrm{K}^{+}\right)$. The speciation is estimated by describing the dissociation equilibrium acids with the following equations

$$
A H \rightleftharpoons H^{+}+A^{-} \quad\left(K_{1}\right)
$$

in which the dissociation constant $K_{1}$ is given by

$$
K_{1}=\frac{\left[H^{+}\right]\left[A^{-}\right]}{[A H]} Y_{1}
$$

where $Y_{1}$ is the quotient of the activity coefficients $\gamma_{j}$,

$$
Y_{1}=\frac{\gamma_{H^{+}} \gamma_{A^{-}}}{\gamma_{A H}}
$$

for a monoacid such as acetic acid. In the case of carbonate and bicarbonate solutions the following two equilibria have been taken into account

$$
\begin{aligned}
& A H_{2} \rightleftharpoons H^{+}+A H^{-} \quad\left(K_{2}\right) \\
& A H^{-} \rightleftharpoons H^{+}+A^{2-} \quad\left(K_{3}\right)
\end{aligned}
$$

The equilibrium constants $K_{2}$ and $K_{3}$ are given in a way similar to eqs. (2) and (3). One has,

$Y_{2}=\frac{\gamma_{H^{+}} \gamma_{A H^{-}}}{\gamma_{A H_{2}}} \quad$ and $\quad Y_{3}=\frac{\gamma_{H^{+}} \gamma_{A^{2-}}}{\gamma_{A H^{-}}}$

Moreover, in the case of sodium carbonate solutions, in a second step we took also into account an association reaction between the cation $\mathrm{Na}^{+}$and the bivalent carbonate anion

$N a^{+}+A^{2-} \rightleftharpoons N_{a} A^{-} \quad\left(K_{A}\right)$

In the same way, $Y_{A}=\gamma_{N a A^{-}} /\left(\gamma_{N a^{+}} \gamma_{A^{2-}}\right)$. Our approach is similar to that used by Apelblat to describe the speciation of organic acids and their salts in dilute solutions [70], except that the individual activity coefficients have been calculated within the MSA theory. The expressions used to calculate these activity coefficients can be found in the appendix of a previous article [71]. The chemical equilibria were solved by iterations. In a first step, the activity coefficients are neglected. An initial estimate of the concentrations of the various species is obtained and a first estimate of the activity coefficients is deduced from these concentrations. Then, these activity coefficients are used to evaluate the quotients $Y_{1}$ and $Y_{2}$. A new estimate of the concentrations of the various species is obtained by taking into account these quotients in the chemical equilibria. Then, a new estimate of the activity coefficients can be deduced, etc. The iterative calculation is stopped when the accuracy of the concentrations values is estimated sufficient. 


\subsection{Description of the conductivity in non-dilute solutions}

The theoretical description of the electrical conductivity used here is based on Onsager's continuity equations [50]. The specific conductivity of the solution is given by the following formula:

$\chi=\frac{10^{3} e^{2} \mathcal{N}_{A}}{k_{B} T} \sum_{i} c_{i} z_{i}^{2} D_{i}\left(1+\frac{\delta k_{i}}{k_{i}}\right)\left(1+\frac{\delta v_{i}^{e l}}{v_{i}^{o}}\right)$

where $c_{i}$ is the molar concentration of component $i, \mathcal{N}_{A}$ is Avogadro number, $k_{B}$ the Boltzmann constant, $T$ the temperature, $z_{i}$ the valency of ion $i$ and $D_{i}$ its diffusion coefficient at infinite dilution. In this calculation two contributions are responsible for deviations from ideality : $\delta k_{i}$ is the relaxation correction on the electric force $k_{i}=z_{i} e E$ acting on species $i$ and $\delta v_{i}^{e l} / v_{i}$ is the relative hydrodynamic or electrophoretic correction. We have used an electrophoretic correction deduced previously from the distribution functions given by the MSA theory for ions of different radii [72]. Initially, the relaxation contribution was calculated, for binary electrolyte only, from the Fuoss-Onsager continuity equations, using also the ion distribution function at equilibrium given by the MSA [24, 25, 28]. For an electrolyte containing more than two types of ions the Fuoss-Onsager continuity equation system is more complicated to solve. Onsager and Kim gave a general mathematical method for solving these equations for any number of charged species. They deduced a limiting law (in the square root of the concentrations) [73]. Extended laws, applicable to less diluted solutions, have been also developed [53-55]. By introducing the MSA instead of the Debye and Hückel equilibrium distribution functions, in the Onsager-Kim formalism, an expression of the relaxation contribution applicable to mixtures at higher concentrations was obtained [34]. Subsequently simplified expressions derived from this work was succesfully used to describe simple electrolyte mixtures [35].

We have used here the same expressions as in this latter article. For convenience the equivalent conductivity $\Lambda^{e}$ was used for a binary electrolyte. It is defined by the specific conductivity $\chi$ divided by the equivalent concentration $c_{e q}=c_{1}\left|z_{1}\right|=c_{2}\left|z_{2}\right|$.

$\Lambda^{e}=\lambda_{1}+\lambda_{2} \quad$ with $\quad \lambda_{i}=\frac{10^{3} e^{2} \mathcal{N}_{A}}{k_{B} T}\left|z_{i}\right| D_{i}\left(1+\frac{\delta k_{i}}{k_{i}}\right)\left(1+\frac{\delta v_{i}^{e l}}{v_{i}^{o}}\right)$

In a dissociated binary electrolyte the relative relaxation forces are equal $\left(\delta k_{1} / k_{1}=\right.$ $\delta k_{2} / k_{2}$ ) [50]. Thus, for dissociated binary electrolytes, the contributions $\lambda_{1}$ and $\lambda_{2}$ to the conductivity due to cations and anions can be easily calculated. As a result, the transport number $t_{i}=\lambda_{i} / \Lambda^{e}$ turn out to be independent of the relaxation forces. For symmetric binary electrolyte the molar conductivity $\Lambda$ was also used. It is defined by the specific conductivity divided by the total molar concentration $\mathrm{C}: \Lambda=\chi / C$.

On the other hand, when the electrolyte contains more than 2 types of ions, the relative relaxation forces are no longer equal. Then, individual contributions to the conductivity are more difficult to evaluate. As all of our conductivity calculations are done by associating the MSA theory with Fuoss-Onsager transport theory, the result of these calculations will be called 'MSA-Transport' calculation in the figures of the article. 


\section{Description of the supporting electrolyte}

\subsection{Sodium acetate and $\mathrm{NaOH}$ solutions}

We first study the chemical equilibria and conductivity of sodium acetate in solution. To correctly describe the proportion of the various species, it is necessary to take into account the acid-base reaction which controls the dissociation of acetic acid. Sodium acetate can be seen as resulting from the reaction of acetic acid with sodium hydroxide. The amount of hydroxide ion is related to the amount of hydronium ion.

The solution of the chemical equilibria leads to the concentration of the various chemical species present. For this electrolyte, in the range studied $\left(10^{-4}-1 \mathrm{M}\right)$, the amount of hydronium ions is negligible. Then, the conductivity may be calculated by considering only the contributions of the sodium, acetate and hydroxide ions. To do this, we used the literature values for the limiting conductivity at infinite dilution $\lambda^{o}$ for these ions [74]. Moreover, one needs to assign a size to each of these ions in the MSA-transport calculation. In the case of the sodium ion, its size has been previously determined in order to best describe the conductivity of $\mathrm{NaCl}$ solution. The size of the hydroxide ion was assessed by describing the conductivity of $\mathrm{NaOH}$ solution. It was noted that the adjustment of the measured conductivity of $\mathrm{NaOH}$ is better when the size of the $\mathrm{OH}^{-}$ion is very small. However, the question arises as to what is the smallest size that remains realistic. For simple ions like halide, alkali and alkaline earth ions, it is generally assumed that the smallest size they can have is their crystallographic radius. Unfortunately, for molecular ions, i.e. composed of different atoms linked together, it is more difficult to assign to each of these ions a crystallographic radius. Thus, in order to have an element of comparison, we have also studied the variation of conductivity in aqueous solutions of $\mathrm{NaF}$ at $25 \mathrm{C}$. Assuming that this salt is totally dissociated and neglecting the hydrolysis of fluoride ions, it was found that by taking for the ion $\mathrm{F}^{-}$a radius $\mathrm{R}=1.36 \stackrel{\circ}{\mathrm{A}}$, we obtain a reasonable agreement between the calculation and the experimental data. This value is precisely its crystallographic radius found in literature [74]. Then, for comparison, we evaluated the value of the conductivity of $\mathrm{NaOH}$ as a function of its concentration, considering that the $\mathrm{OH}^{-}$ion has a radius equal to either that of the $\mathrm{Cl}^{-}$ion $(\mathrm{R}=1.81 \AA)$, or to that of the $\mathrm{F}^{-}$ion. The agreement is better with the smaller radius. As a result, the study of the conductivity of sodium acetate solution leads us to assign a size to the acetate ion. Since the hydroxide and acetate ions are sometimes deemed to be complexing ions, we wanted to know if the profile of the observed conductivities, reflected an association between these ions and the sodium cations. For comparison, we also described the conductivity of sodium perchlorate solution. The perchlorate ion is considered to be non-complexing with simple monovalent cations and solutions of this ion are often used to adjust the ionic strength in an electrophoresis experiment. In the same way, the size of the perchlorate ion was determined in order to best describe the observed evolution of the conductivity of these solutions. The set of limiting conductivities $\lambda^{\circ}$ and ion sizes used in this study are collected in Table 1. The calculated and experimental conductivities of these solutions are presented in Figure 1. Our model describes quite well the conductivities of perchlorate and sodium acetate solutions. However, for sodium acetate, the experimental points deviate slightly more from the calculated curve for concentrations of the order of $1 \mathrm{M}$. 
Our model describes well the experimental conductivity of sodium hydroxide at low concentrations (less than $0.1 \mathrm{M}$ ). On the other hand, the agreement between the calculated and observed values is not as good at high concentrations.

This can be expected in view of the fact that the conductivity of the hydroxide ion (in the same way as that of the hydronium ion) is not mainly due to a simple migration process, as for the other ions, but rather to a mechanism of charge transfer between the molecules without moving molecules. Our model, like the models of Debye and Onsager, considers ions as Brownian particles. It is therefore not adapted to describe the migration of these particular ions. Nevertheless, it allows to describe the conductivity of solutions containing these ions at low concentrations. Therefore, when the $\mathrm{pH}$ is not too high, a quantitative description of the conductivity of solution containing salts of weak acids seems possible.

It results from the determination of the quantities of the various species in equilibrium that the major species are only the acetate and sodium ions, as soon as the concentration is greater than $10^{-3} \mathrm{M}$. These ions mainly determine the conductivity. Thus, at high concentration, this solution is very similar to a binary electrolyte. The complexation equilibria involved in describing this solution appear simple in comparison with those governing the proportion of the various charged species in most buffer solutions usually used. Indeed, in many buffer solutions several acid-base equilibria exist. Consequently, the number of different charged species are more important. When more than two ionic species contribute significantly to the conductivity, the use of the expressions devoted to any mixtures become essential. Then, as a second example of the application of our theoretical approach, we now describe the solutions of alkali hydrogen carbonate and carbonate ions. In this example the number of species contributing to the conductivity is greater. This salt does not behave like a binary electrolyte.

\subsection{Carbonate and hydrogen carbonate salts solutions}

Next we have studied the conductivities of binary salts in which the carbonate and bicarbonate ions are present. As explained in the theoretical part on speciation, in the cases of the carbonate and bicarbonate ions we need to consider the chemical equilibria linking these ions with the $\mathrm{pH}$ and the quantity of carbonic acid present in the solution.

We have tried in a first step to describe two types of solutions of hydrogen carbonate, namely $\mathrm{NaHCO}_{3}$ and $\mathrm{KHCO}_{3}$. For these solutions the calculation of the proportions of the various species leads to a greater amount of $\mathrm{HCO}_{3}^{-}$than $\mathrm{CO}_{3}^{2-}$ ions. When the solution is concentrated the $\mathrm{Na}^{+}$and $\mathrm{HCO}_{3}^{-}$ions mainly contribute to the conductivity. Unlike acetate solutions, the conductivity of hydrogen carbonate solutions seems to have been less measured. At low concentrations the conductivity of $\mathrm{NaHCO}_{3}$ was measured by Kendall [63] and that of $\mathrm{KHCO}_{3}$ by MacInnes and Shedlovsky [37]. These last measurements made it possible to determine the dissociation constant of acetic acid and the limiting conductivity of the bicarbonate ion. In our calculations the value of the limiting conductivity of the bicarbonate ion determined by these authors was used. On the other hand, extrapolation to low concentrations of the measured conductivity of the $\mathrm{NaHCO}_{3}$ solutions leads unfortunately to a larger value of the limiting conductivity of the $\mathrm{HCO}_{3}^{-}$

ion. So, we did not continue the study of the conductivity of $\mathrm{NaHCO}_{3}$. Again, to describe 
the conductivity of $\mathrm{KHCO}_{3}$, we have used the value for the diameter of the potassium ion obtained previously in the fitting of the conductivities of $\mathrm{KCl}$ and $\mathrm{KBr}$ [35]. Then, we have determined an effective radius for the hydrogen carbonate ion which best describes the data for the conductivity of $\mathrm{KHCO}_{3}$ solutions. The conductivity of this salt is shown in Figure 1. The evolution of the conductivity of this salt with concentration is similar to that observed for sodium acetate. Likewise, the experimental points deviate slightly from the calculated curve for concentrations of the order of $1 \mathrm{M}$.

Next, we tried to describe $\mathrm{Na}_{2} \mathrm{CO}_{3}$ solutions. For these solutions both the carbonate ions and the hydrogen carbonate ions contribute to the conductivity. The amount of hydroxide ion is also greater. At low concentrations the amount of $\mathrm{HCO}_{3}^{-}$ions is larger than that of $\mathrm{CO}_{3}^{2-}$ ions. At high concentrations the amount of $\mathrm{CO}_{3}^{2-}$ ions becomes greater than that of $\mathrm{HCO}_{3}^{-}$ions. Consequently, at low concentrations, it is found that the $\mathrm{OH}^{-}$and $\mathrm{HCO}_{3}^{-}$ions are the anions that contribute most to the conductivity. Eq. (8) allows us to calculate the specific conductivity $\chi$ of $\mathrm{Na}_{2} \mathrm{CO}_{3}$ solutions from the individual contributions of the predominant ions: $\mathrm{Na}^{+}, \mathrm{CO}_{3}^{2-}, \mathrm{HCO}_{3}^{-}$and $\mathrm{OH}^{-}$. The equivalent conductivity $\Lambda_{e}$ of the solution was calculated by dividing the specific conductivity $\chi$ by the concentration of $\mathrm{Na}^{+}$ions. As noted by Monk [41], this conductivity can be decomposed into the sum of the of partial conductivities of three salts: $\mathrm{Na}_{2} \mathrm{CO}_{3}, \mathrm{NaHCO}_{3}$ and $\mathrm{NaOH}$, multiplied by the proportion of each salt as,

$\Lambda_{e}=\frac{\left[\mathrm{OH}^{-}\right]}{\left[\mathrm{Na}^{+}\right]}\left(\lambda_{\mathrm{Na}}+\lambda_{\mathrm{OH}}\right)+\frac{\left[\mathrm{HCO}_{3}^{-}\right]}{\left[\mathrm{Na}^{+}\right]}\left(\lambda_{\mathrm{Na}}+\lambda_{\mathrm{HCO}_{3}}\right)+\frac{2\left[\mathrm{CO}_{3}^{2-}\right]}{\left[\mathrm{Na}^{+}\right]}\left(\lambda_{\mathrm{Na}}+\lambda_{\mathrm{CO}_{3}}\right)$

In addition, Monk assumed that each of the partial conductivities, $\left(\lambda_{N a}+\lambda_{O H}\right)$ for $\mathrm{NaOH}$, $\left(\lambda_{N a}+\lambda_{\mathrm{HCO}_{3}}\right)$ for $\mathrm{NaHCO}_{3}$ and $\left(\lambda_{N a}+\lambda_{C O_{3}}\right)$ for $\mathrm{Na}_{2} \mathrm{CO}_{3}$, could be represented by the theoretical expression accounting for the corresponding binary salt. With this approximation the partial contributions of these salts to the equivalent conductivity $\Lambda_{e}$ could be calculated. Furthermore, when the conductivity of the binary electrolyte is represented by an expansion in powers of the square root of the salt concentration, Monk assumed that the partial conductivities involved in the mixture can be represented by the same expansion but replacing the salt concentration with the ionic strength $I$ of the solution $\left(I=1 / 2 \sum_{i} c_{i} z_{i}^{2}\right)$. With this approximation, the calculated contributions of $\mathrm{NaOH}$ and $\mathrm{NaHCO}_{3}$ can be subtracted from the equivalent conductivity $\Lambda_{e}$ given by eq. (10). One then obtains the partial contribution $\left(\lambda_{N a}+\lambda_{C O_{3}}\right)$ of the salt $\mathrm{Na}_{2} \mathrm{CO}_{3}$. The limiting conductivity of the $\mathrm{CO}_{3}^{2-}$ ion was determined previously using this method.

We note that this approximate expression does not satisfy the limiting law in the square root of the concentration given by Onsager $[50,73]$. In our study we did not use this approximation. We computed the set of individual contributions from expressions established using the Onsager and Kim formalism for ionic mixtures [35]. At low concentrations, these expressions tend toward the limiting law in square root of the concentration. The conductivity calculated using this formalism is compared with experimental data $[41,75]$ in Figure 2. Taking into account that the previous determination of the limiting conductivity of the $\mathrm{CO}_{3}^{2-}$ ion was made with an approximate law, we again determined the value of this limiting conductivity in order to obtain the best agreement between the calculated curve and the experimental data at low concentrations. We found 
$\lambda\left(\mathrm{CO}_{3}^{2-}\right)=6.83 \mathrm{mS} \mathrm{m}^{2} \mathrm{~mol}^{-1}$ instead of $6.93 \mathrm{mS} \mathrm{m}^{2} \mathrm{~mol}^{-1}$ previously determined. Since the conductivity depends on the proportion of the various ions present, it is interesting to study their relative contributions. As the solution is neutral overall, we have chosen to represent the contribution of the various species in term of the three intervening salts of eq. (10). Then, we have also shown in Figure 2 the relative contributions to the conductivity of the $\mathrm{NaOH}, \mathrm{NaHCO}_{3}$ and $\mathrm{Na}_{2} \mathrm{CO}_{3}$ couples (the dashed or dotted blue, green and red curves, respectively). When the concentration tends to zero, the contributions of $\mathrm{NaOH}$ and $\mathrm{NaHCO}_{3}$ appear larger than the contribution of $\mathrm{Na}_{2} \mathrm{CO}_{3}$. At low concentrations the three couples of ions contribute to the observed equivalent conductivity. When the concentration is increased, the contributions of $\mathrm{NaOH}$ and $\mathrm{NaHCO}_{3}$ become less and less important. Our model correctly describes the measured conductivity, especially at low concentrations.

It may be noticed that in order to inprove the agreement between the measurements and the calculated curve, the size of the $\mathrm{CO}_{3}^{2-}$ ion could be lowered, which would have decreased the calculated values. However, the radius chosen for this ion already seems rather small. As an alternative, it can be assumed that the reduction of the conductivity at high concentrations may be due to the formation of complexes between anions and the $\mathrm{Na}^{+}$cations. For simplicity we considered only one type of complex, namely the ion $\mathrm{NaCO}_{3}^{-}$. The $\mathrm{CO}_{3}^{2-}$ ion being the most charged anion, it was assumed that only this ion forms complexes in this solution. To describe the influence of the $\mathrm{NaCO}_{3}^{-}$ion, it was assumed that its limiting conductivity and its size were equals to that of $\mathrm{HCO}_{3}^{-}$, to limit the number of additional parameters. Only the association constant remains to be determined in order to evaluate the theoretical conductivity. Taking an association constant $K_{A}=1.5 \mathrm{~L} \mathrm{~mol}^{-1}$ we obtain the curve represented in Figure. 2. The value obtained for the association constant is rather low in comparison with those quoted in the literature [76]. As expected, it is observed that the decay of the calculated conductivity is more important than that obtained without association. As a result, the agreement between the calculated curve and the experimental data is better at high concentrations.

\subsection{A dissociated acid in solution : $\mathrm{HCl}$}

The solutions we have described so far are all either basic or neutral. A satisfactory description of the conductivity of $\mathrm{NaOH}$ solutions has been obtained for concentrations below $0.1 \mathrm{M}$. Otherwise, in order to minimize hydrolysis phenomena it is sometimes useful to dissolve some electrolytes in acidic solutions. Dissolution may also lead to acidification of the solution. It is therefore interesting to study also the ability of our theory to describe somewhat concentrated acidic solutions. For this purpose, we have examined the conductivity of hydrochloric acid solutions. In our previous study, we considered various solutions of alkali chloride ions. The size of the chloride ion was deduced by adjusting the conductivities of these salts. It is therefore this acid that we have chosen to describe, insofar as the only parameter yet to be described is the effective size of the hydronium ion. Moreover, many experimental data are available. In addition, the ionic transport numbers have also been determined experimentally for these solutions. So we calculated the total conductivity and the individual conductivities of the cations and anions in these 
solutions.

A comparison between experimental and theoretical variations is given in the upper curve of Figure 3. It is found that the effective size of the proton must be extremely small to increase the agreement between calculated and measured conductivities. In order to keep a realistic size parameter for this ion, we chose to assign it the same size as the lithium ion determined in the previous study. As a result, a good description of the total conductivity is obtained up to a concentration of the order of $0.2 \mathrm{M}$. Beyond this concentration, the experimental conductivity decreases faster than the calculated one. The other two curves represent the conductivity due to the proton (in red) and that due to the chloride ion (in blue). It is noted that the latter is well represented up to $1 \mathrm{M}$, whereas that due to the proton ceases to be well described by our model beyond $0.2 \mathrm{M}$. The limitation observed for the conductivity due to protons is consistent with what we could expect considering the evolution observed for the total calculated conductivity. The fact that the conductivity due to chloride ions remains well described at higher concentrations is very promising. Despite the intrinsic inadequacy of our model to account for the evolution of the value of proton mobility with concentration, this suggests that the variation in anion mobility remains well depicted.

Despite the peculiar nature of proton conduction, it is questionable whether the various ways of improving the description of the solutions presented earlier in this article are suitable to give rise an improvement in the description of acidic solutions. In the previous section we considered two different ways to inprove the agreement between the theoretical description and the experimental conductivity data:

- For fully dissociated electrolytes, the only adjustable parameter wass the size of the cation. When the value of the ion size used in the model decreases, the calculated conductivity decreases.

- On the other hand, when the ions of a symmetrical electrolyte can associate to form neutral pairs, the amount of charge carriers (non-associated ions) decreases and the conductivity decreases.

In the case of $\mathrm{HCl}$ solution, we have already taken a small size for the proton. Choosing to further decrease the size of the proton might seem unrealistic. So, although this electrolyte is considered to be completely dissociated, one could consider an association between the $\mathrm{Cl}^{-}$anions and the $\mathrm{H}^{+}$cations to further decrease the calculated conductivity. Thus, if the fraction of cation or free anion is denoted by $\alpha$, the equivalent conductivity is given by

$\Lambda^{e}=\alpha\left(\lambda_{1}+\lambda_{2}\right)$

with $\lambda_{1}$ and $\lambda_{2}$, functions of $\alpha$ and the concentration. When the electrolyte is associated, $\alpha$ is less than 1 and the conductivity decreases. The sharp decrease in the conductivity of acetic acid can be explained by taking into account such an association. However in the case of $\mathrm{HCl}$ solution, besides the fact that there is no experimental fact which indicates that this electrolyte is associated (at least in this range of concentration), the fact of taking into account such an association would also modify the individual conductivities of the two types of ions. However, without association the conductivity due to chloride 
ions remains well described at high concentrations. Taking into account an association would improve probably the description of the conductivity due to the protons but would at the same time impair the description of the conductivity due to chlorides. A similar impaired description of the individual conductivities should be expected also for solutions obtained by dissolving an electrolyte containing $\mathrm{Cl}^{-}$anions in a solution of $\mathrm{HCl}$. As a result, considering an association between anions and cations is not suitable to describe the observed decrease in the conductivity of acids such $\mathrm{HCl}$ in solution. Finally, the joint modeling of the total conductivity but also of the individual conductivities (using the transport numbers), provides a better understanding of the solutions studied.

\section{Conclusion}

Generally the theoretical conductivity models are applied only to aqueous solutions of small monoatomic ions such as alkali or alkaline earths halides. Then, this has been done previously for binary salts and mixtures with the model used here. Considering only interactions of hard spheres at short distances and coulombic interactions at great distances the transport properties of these simple salts are well described by our model. However, all the solutions are not so simple. The alkaline halides salts appear to be partially dissociated in organic solution. Similarly, 2-2 electrolytes such as $\mathrm{MgSO}_{4}$ would form ion pairs in aqueous solution. This association seems to be mainly of electrostatic nature and can be calculated using an association constant deduced from the Bjerrum theory or its extensions.

Often other interactions need to be considered. Associations of a chemical nature such as those leading to acid-base equilibria should be considered to properly describe the properties of the solutions presented in this article. For sodium acetate and potassium bicarbonate solutions we have assumed that there is no additional anion-cation association so as not to complicate the model further. In the same way, in a first step, we did not consider this type of association to describe the conductivity of $\mathrm{Na}_{2} \mathrm{CO}_{3}$ solution. Knowing the limiting conductivities the only parameters of the model are the sizes of the anions (the radii of the cations having been determined in previous studies). A satisfactory description of the experimental conductivities has thus been obtained for these solutions. In the case of $\mathrm{Na}_{2} \mathrm{CO}_{3}$ solutions the limiting equivalent conductivity of the $\mathrm{CO}_{3}^{2-}$ ion was previously determined from the analysis of the experimental data at low concentrations using an approximate expression of the theoretical conductivity. However, this expression does not satisfy Onsager's limiting law. We have therefore more precisely determined this limiting conductivity with the help of our model which tends towards the limiting law of Onsager at low concentrations. The limiting conductivity determined in this way is a little bit lower than that previously determined using the less rigorous approach. As noted previously, the contributions of $\mathrm{OH}^{-}$and $\mathrm{HCO}_{3}^{-}$ions are important at low concentrations. Contrary to what one would have if $\mathrm{Na}_{2} \mathrm{CO}_{3}$ was totally dissociated, extrapolation to low concentrations does not simply lead to to the sum of infinite dilution conductivities of

$\mathrm{Na}^{+}$and $\mathrm{CO}_{3}^{2-}$. This clearly highlights the need to use a theory that satisfies the limiting law for electrolyte mixtures. In a second step, in order to improve the agreement between the theory and the experimental data of the $\mathrm{Na}_{2} \mathrm{CO}_{3}$ solution conductivity, we took into 
account the formation of the $\mathrm{NaCO}_{3}^{-}$species. For simplicity, we assumed that the species $\mathrm{NaCO}_{3}^{-}$had the same limiting conductivity and the same size as the $\mathrm{HCO}_{3}^{-}$ion. These two quantities could be determined together with the value of the association constant, which would certainly improve the agreement between modeling and experimental data. However it must be noted that there is very few experimental data at high concentrations. Often a single set of data for a given salt. It is therefore difficult to judge the accuracy of these data. As a result, given the fairly good description of the data already obtained, we have not sought to further refine the determination of the parameters related to the complexes formed.

In order to characterize all the ions that may be present, we have also studied the ability of our theory to describe some strong alkaline or acid solutions. Our model correctly describes the experimental conductivity of $\mathrm{NaOH}$ and $\mathrm{HCl}$ solutions at low concentrations (less than $0.1 \mathrm{M}$ ). The range of reliability is narrower than that of simple electrolyte solutions. Nevertheless we have shown for $\mathrm{HCl}$ solutions that the conductivity of the $\mathrm{Cl}^{-}$ counterions remains well described. This is encouraging for future applications of our model to describe simple electrolytes dissolved in acidic solutions.

In order to extend this study, other electrolyte solutions could be considered. First, having characterized solutions of sodium acetate or carbonate, for which the complexation between cations and anions appear to play a minor role, then we may be interested in solutions containing more charged cations, such as magnesium, for which the association with the anions may have a greater influence on the conductivity of the solution. On the other hand, other ionic solutions used as buffers can be considered with the same approach. For example it would be interesting to be able to describe the variation of the conductivity of concentrated solutions of salts containing phosphate or citrate anions. Finally, the description of acidic solutions that we presented, may be useful during the analysis of the conductivity of salts, possibly containing divalent or trivalent cations, dissolved in acidic solutions.

\section{Acknowledgment}

This work is in the continuity of a study that was started, initially with the financial support of GDR Paris. The first meeting of the authors, at the beginning of this study, was initiated by P. Turq. We spare a thought for Pierre Turq who passed away, a good friend and an excellent scientist. 


\section{References}

[1] C. Ambard, A. Delorme, N. Baglan, J. Aupiais, ; F. Pointurier and C. Madic, Radiochim. Acta, 93 (2005) 665-673.

[2] J. Petit, V. Geertsen, C. Beaucaire and M. Stambouli, J. Chromatogr. A, 1216 (2009) 4113-4120.

[3] G. Alvarez-Llamas, M. R. Fernandez delaCampa, and A. Sanz-Medel, Trends Anal. Chem. 24 (2005) 28-33.

[4] J. E. Sonke and V. J. M. Salters, J. Chromatogr. A, 1159 (2007) 63-74.

[5] J. Aupiais, A. Delorme and N. Baglan, J. Chromatogr. A, 994 (2003) 199-206.

[6] N. Wu, W. J. Horvath, P. Sun and C. W. Huie, J. Chromatogr., 635 (1993) 307-312.

[7] J. Havel, P. Janos and P. Jandik, J. Chromatogr. A, 745 (1996) 127-134.

[8] M. Karbowiak, B. Fourest, S. Hubert and C. Moulin, Radiochimica Acta, 91 (2003) 505-512.

[9] V. Philippini, J. Aupiais, T. Vercouter and C. Moulin, Electrophoresis, 30 (2009) 3582-3590.

[10] S. Topin, J. Aupiais, N. Baglan, T. Vercouter, P. Vitorge and P. Moisy, Anal. Chem., 81 (2009) 5354-5363

[11] S. Topin, J. Aupiais and P. Moisy, Electrophoresis, 30 (2009) 1747-1755

[12] D. Li, S. Fu and C. A. Lucy, Anal. Chem., 71 (1999) 687-699.

[13] A. Jouyban and E. Kenndler, Electrophoresis, 27 (2006) 992-1005.

[14] S. S. Bahga, M. Bercovici and J. G. Santiago, Electrophoresis, 31 (2010) 910-919.

[15] S. A. Allison, H. Pei, S. Baek, J. Brown, M. Y. Lee, V. Nguyen, U. T. Twahir and H. Wu, Electrophoresis, 31 (2010) 920-932.

[16] C. Y. Mou, T. S. Thatcher and J. L. Lin, J. Chem. Phys., 79 (1983) 957.

[17] C. Engel-Herbert, D. Kremp and J. Töwe, J. Solution Chem., 19 (1990) 225-246.

[18] O. Bernard, W. Kunz, P. Turq and L. Blum, J. Phys. Chem., 96 (1992) 398-403.

[19] J.-F. Dufrêche, O. Bernard, P. Turq, A. Mukherjee and B. Bagchi, Phys. Rev. Lett., 88 (2002) 123-133.

[20] J.-F. Dufrêche, M. Jardat, P. Turq and B. Bagchi,J. Phys. Chem. B, 112 (2008) 10264-10271.

[21] O. Bernard, T. Cartailler, P. Turq and L. Blum, J. Mol. Liq., 73-74 (1997) 403-411. 
[22] J.-F. Dufrêche, O. Bernard and P. Turq, J. Mol. Liq., 96-97 (2002) 123-133. J.-F. Dufrêche, O. Bernard and P. Turq,J. Chem. Phys., 116 (2002) 2085-2097.

[23] W. Ebeling, R. Feistel and R. Sänding,J. Solution Chem., 8 (1979) 53-82.

[24] W. Ebeling and J. Rose, J. Solution Chem., 10 (1981) 599-609.

[25] W. Ebeling and M. Grigo, J. Solution Chem., 11 (1982) 151-167.

[26] A. R. Altenberger, J. Phys. A , 14 (1981) 957

[27] A. R. Altenberger and H. L. Friedman, J. Chem. Phys., 78 (1983) 4162.

[28] O. Bernard, W. Kunz, P. Turq and L. Blum, J. Phys. Chem., 96 (1992) 3833-3840

[29] P. Turq, L. Blum, O. Bernard and W. Kunz, J. Phys. Chem., 99 (1995) 822-827.

[30] A. Anderko and M. M. Lencka, Ind. Eng. Chem. Res., 36 (1997) 1932-1943;

[31] P. Wang, A. Anderko and R. D. Young, Ind. Eng. Chem. Res., 43 (2004) 8083-8092

[32] S. Durand-Vidal, O. Bernard and P. Turq, J. Phys. Chem., 100 (1996) 17345-17350.

[33] J.-F. Dufrêche, O. Bernard, S. Durand-Vidal and P. Turq, J. Phys. Chem. B, 109 (2005) 9873-9884.

[34] S. Van Damme and J. Deconinck, J. Phys. Chem. B, 111 (2007) 5308-5315

[35] G. M. Roger, S. Durand-Vidal, O. Bernard and P. Turq, J. Phys. Chem. B, 113 (2009) 8670-8674.

[36] S. Rossy-Delluc, T. Cartailler, P. Turq, O. Bernard, N. Morel-Desrosiers, J.-P. Morel and W. Kunz, J. Phys. Chem., 97 (1993) 5136-5140.

[37] D. A. MacInnes and T. Shedlovsky, J. Am. Chem. Soc., 54 (1932) 1429-1438

[38] T. Shedlovsky and D. A. MacInnes, J. Am. Chem. Soc., 57 (1935) 1705-1710

[39] B. Saxton and L. S. Darken, J. Am. Chem. Soc., 62 (1940) 846-852

[40] L. S. Darken, J. Amer. Chem. Soc., 63 (1941) 1007

[41] C. B. Monk, J. Chem. Soc., (1949) 429-431

[42] S. David Klein and R. G. Bates, J. Solution Chem., 9 (1980) 289 -292

[43] A. Apelblat and J. Barthel, Z. Naturforsch. A, 46 (1991) 131.

[44] E. N. Tsurko, R. Neueder, J. Bartheland A. Apelblat J Solution Chem., 28 (1999) 973-999

[45] M. Bešter-Rogač, M. Tomšič, J. Barthel, R. Neueder and A. Apelblat, J. Sol. Chem., 31 (2002) 1-18 
[46] G. H. Zimmerman and R. H. Wood, J. Sol. Chem., 31 (2002) 995-1017

[47] A. D. Pethybridge, J. D. R. Talbot and W. A. House, J. Sol. Chem., 35 (2006) 381-393

[48] M. Bončina, A. Apelblat and M. Bešter-Rogač, J. Chem. Eng. Data, 55 (2010) 19511957

[49] A. Apelblat and M. Bešter-Rogač, J. Mol. Liq., 247 (2017) 397-402.

[50] L. Onsager and R. M. Fuoss, J. Phys. Chem., 36 (1932) 2689-2778

[51] R. M. Fuoss and L. Onsager, J Phys. Chem., 61 (1957) 668-682.

[52] E. Pitts, Proc. Roy. Soc. A., 217 (1953) 43-70.

[53] J. Quint and A. Viallard, J. Solution Chem., 7 (1978) 533-548

[54] W. H. Lee and R. J. Wheaton, J. Chem. Soc. Faraday Trans. 2,75 (1979) 1128-1145

[55] H. Bianchi and R. Fernandez-Prini, J. Solution Chem., 22 (1993) 557-570

[56] E. Waisman and J. L. Lebowitz, J. Chem. Phys., 52 (1970) 4307 ; J. Chem. Phys. 56 (1972) 3086-3092 ; J. Chem. Phys., 56 (1972) 3093.

[57] L. Blum, Mol. Phys., 30 (1975) 1529. L. Blum and J. S. Høye, J. Phys. Chem., 81 (1977) 1311. K. Hiroike, Mol.Phys., 33 (1977) 1195.

[58] R. Triolo, J. R.Grigera and L. Blum, J. Phys. Chem., 80 (1976) 1858-1861

[59] S. Watanasiri, M. R. Brule and L. L. Lee, J. Phys. Chem., 86 (1982) 292-294

[60] H. R. Corti, J. Phys. Chem., 91 (1987) 686-689

[61] V. Philippini, T. Vercouter, J. Aupiais, S. Topin, C. Ambard, A. Chaussé and P. Vitorge, Electrophoresis, 29 (2008) 2041-2050

[62] C. Claveranne-Lamolère, J. Aupiais, G. Lespes, J. Frayret, E. Pili, F. Pointurier and M. Potin-Gautier, Talanta, 85 (2011) 2504-2510

[63] J. Kendall, J. Am. Chem. Soc., 38 (1916) 1480-1497

[64] K. Park, P. K. Weyl and A. Bradshaw, Nature, 201 (1964) 1283 - 1284

[65] A. Poisson, M. Perie, J. Perie and M. Chemla, J. Solution Chem., 8 (1979) 377-394.

[66] J. A. Sorensen and G. E. Glass, Anal. Chem., 59 (1987) 1594-1597.

[67] J. D. R. Talbot, W. A. House and A. D. Pethybridge, Water Research, 24 (1990) $1295-1304$.

[68] R. B. McCleskey, J. Chem. Eng. Data, 56 (2011) 317-327 
[69] A. Katchalsky, H. Eisenberg and S. Lifson, J. Am. Chem. Soc., 73 (1951) 5889-5890

[70] A. Apelblat, J. Mol. Liq., 95 (2002) 99-145.

[71] G. M. Roger, S. Durand-Vidal, O. Bernard, P. Turq, T.-M. Perger and M. BešterRogač, J. Phys. Chem. B, 112 (2008) 16529-16538.

[72] S. Durand-Vidal, M. Jardat, V. Dahirel, O. Bernard, K. Perrigaud and P. Turq, J. Phys. Chem. B, 110 (2006) 15542-15547.

[73] L. Onsager and S. K. Kim, J. Phys. Chem., 61 (1957) 215-229.

[74] R. A. Robinson and R. A. Stokes, Electrolyte Solutions, Butterworth, London 1959.

[75] T. A. Graber, H. R. Galleguillos, C. Céspedes and M. E. Taboada, J. Chem. Eng. Data, 49 (2004) 1254-1257

[76] P. G. Daniele, C. Foti, A. Gianguzza, E. Prenesti and S. Sammartano, Coordination Chemistry Reviews 252 (2008) 10931107

[77] D. G. Miller, J. Phys. Chem. 70 (1966) 2639-2659.

[78] J. H. Jones, J. Am. Chem. Soc., 67 (1945) 855-857

[79] M. Postler, Coll. Czech. Chem. commun., 35 (1970) 535

[80] V. Sivertz , R. E. Reitmeier and H. V. Tartar, J. Am. Chem. Soc., 62 (1940) 13791382

[81] L. S. Darken and H. F. Meier, J. Am. Chem. Soc., 64 (1942) 621-623

[82] H. S. Harned and B. B. Owen, The physical chemistry of electrolytic solutions, Third Edition, Reinhold Publishing Corporation (NY) 1963, p. 697.

[83] H. M. Rahman, G. Hefter and R. Buchner, J. Phys. Chem. B, 116 (2012) 314-323

[84] P. K. Dasgupta and O. Nara, O. Anal. Chem., 62 (1990) 1117-1122 


\begin{tabular}{||l|c|c|c|c|c|c|c|c||}
\hline & $\mathrm{Na}^{+}$ & $\mathrm{K}^{+}$ & $\mathrm{H}^{+}$ & $\mathrm{ClO}_{4}^{-}$ & Acetate & $\mathrm{OH}^{-}$ & $\mathrm{HCO}_{3}^{-}$ & $\mathrm{CO}_{3}^{2-}$ \\
\hline Radius $(A)$ & 1.17 & 1.70 & 0.80 & 1.81 & 1.81 & $1.81 / 1.36$ & 1.81 & 1.81 \\
$10^{9} \mathrm{D}\left(m^{2} s^{-1}\right)$ & 1.33 & 1.96 & 9.32 & 1.79 & 1.09 & 5.30 & 1.18 & 0.909 \\
$\lambda^{o}\left(\mathrm{mSm}^{2} \mathrm{~mol}^{-1}\right)$ & $5.01^{a}$ & $7.35^{a}$ & $34.98^{a}$ & $6.74^{a}$ & $4.09^{a}$ & $19.92^{a}$ & $4.45^{a}$ & 6.83 \\
\hline
\end{tabular}

${ }^{a}$ Ref. [74].

Table 1: Values of the Radii, Diffusion Coefficients and Limiting Equivalent Conductivities of Each Species. The $\mathrm{Na}^{+}$and $\mathrm{K}^{+}$radii are those previously determined in [35]. The two values of the radius of $\mathrm{OH}^{-}$are those used in Figure 1 (see explanation in the text). The value of limiting equivalent conductivity for $\mathrm{CO}_{3}^{2-}$ was redetermined in this work. 


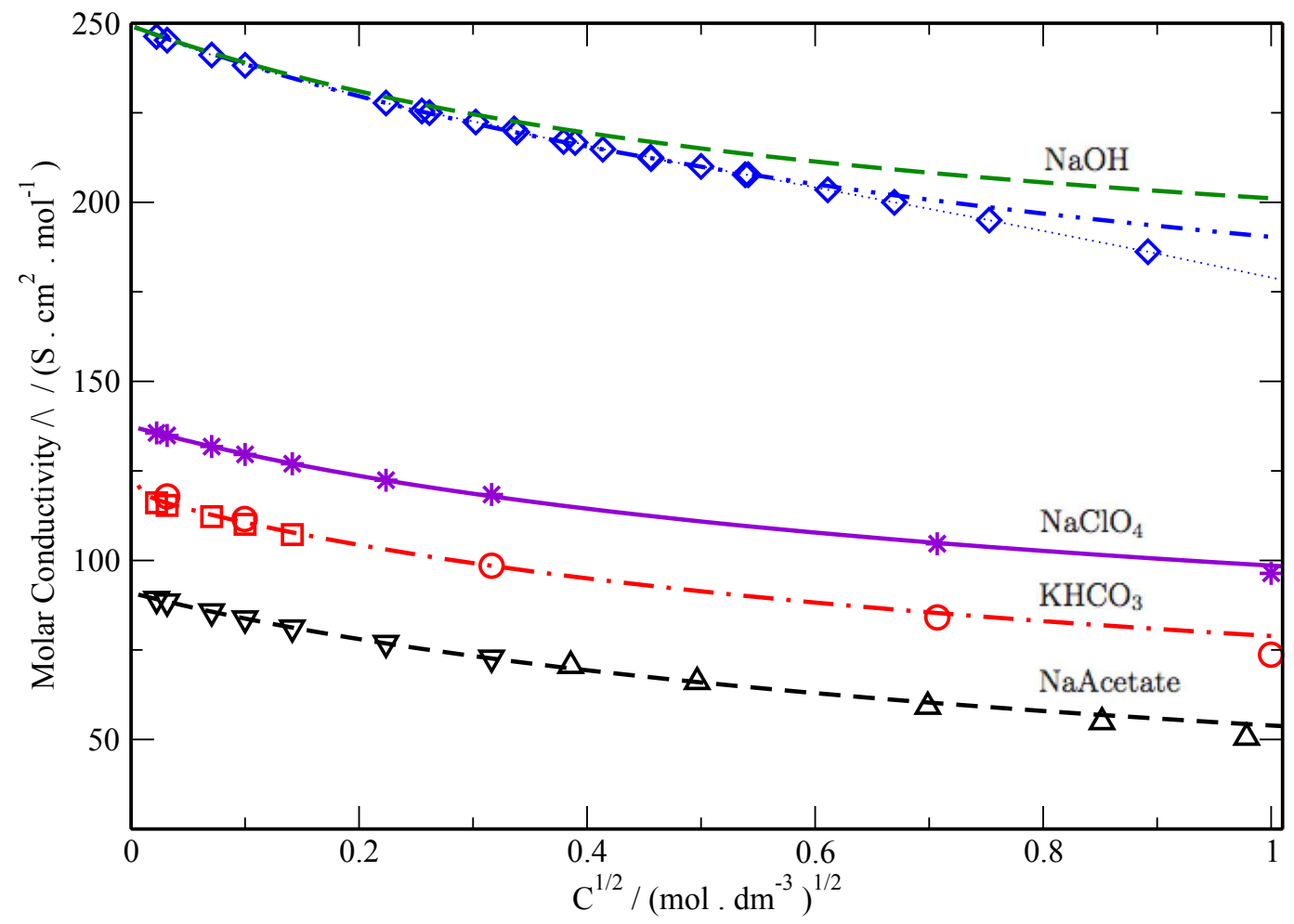

Figure 1: Molar conductivities of solutions at 298.15 K. Upper curve: $\mathrm{NaOH}$ solutions; Experimental data ( [80], [81], diamonds), Calculated curve with $\mathrm{R}_{O H^{-}}=1.81 \AA$ (long dash green line) or with $\mathrm{R}_{\mathrm{OH}^{-}}=1.36 \AA$ (double dot and dashed blue line); The small dots connecting the diamonds are only a guide for the eyes. Second curve: $\mathrm{NaClO}_{4}$ solutions, shifted upwards by $20 \mathrm{~S} . \mathrm{cm}^{2} \cdot \mathrm{mol}^{-1}$; Experimental data ( [78], [79], star), Calculated curve (solid violet line). Third curve: $\mathrm{KHCO}_{3}$ solutions; Experimental data ( [38], squares) and ( [68], circles), Calculated curve (dashed and dotted red line); Lower curve: NaAcetate solutions; Experimental data ( [37], [82], [83], triangles), Calculated curve (dashed black line). 


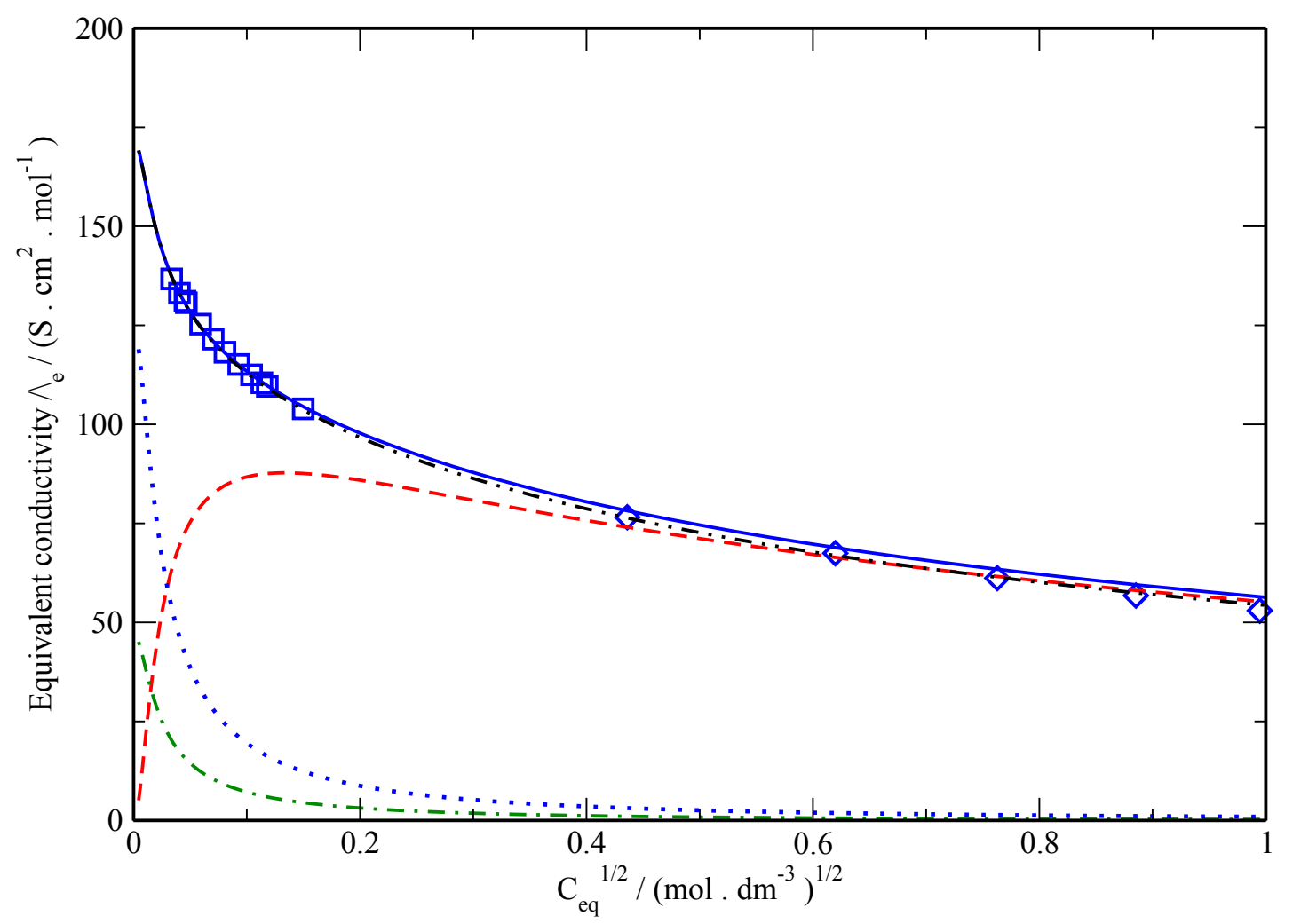

Figure 2: Equivalent conductivity of $\mathrm{Na}_{2} \mathrm{CO}_{3}$ solutions at $298.15 \mathrm{~K}$ : Experimental data ( [41], [75]) (squares and diamonds); MSA-Transport calculations : Contribution from $\mathrm{NaHCO}_{3}$ to the total conductivity (dashed and dotted green line), Contribution from $\mathrm{NaOH}$ to the total conductivity (dotted blue line), Contribution from $\mathrm{Na}_{2} \mathrm{CO}_{3}$ to the total conductivity (dashed red line), Total conductivity of the solutions (solid blue line), Total conductivity of the solution with an association between $\mathrm{Na}^{+}$and $\mathrm{CO}_{3}^{2-}$ (double dotted and dashed black line). 


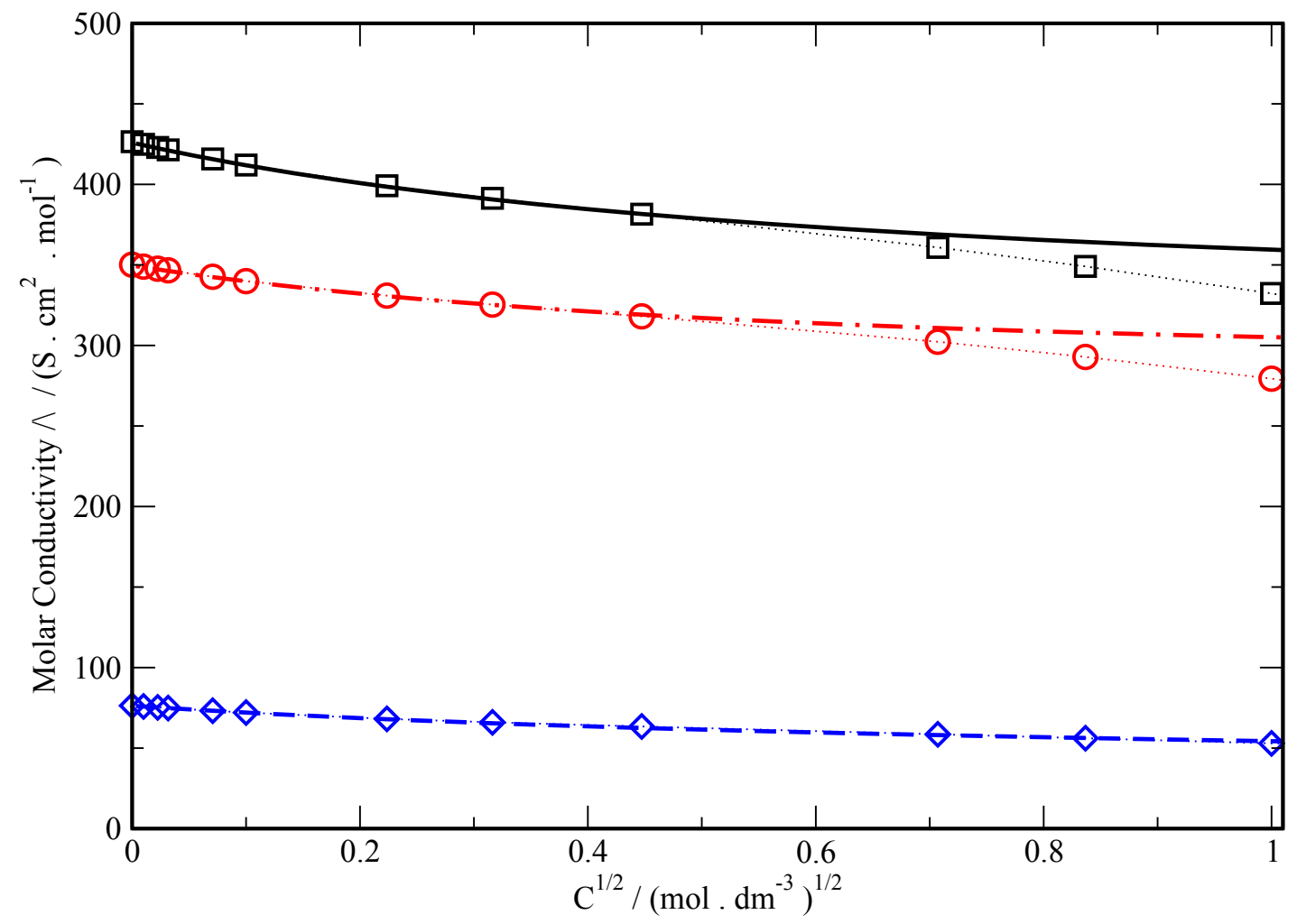

Figure 3: Upper curve : Molar conductivity of $\mathrm{HCl}$ solutions at 298.15 K: Experimental data ( [77] squares), Calculated curve(solid line); Second curve : Contribution of the $\mathrm{H}^{+}$ ions to this molar electrical conductivity : Experimental data ( [77] circles), Calculated curve (dashed and dotted red line); Lower curve : Contribution of the $\mathrm{Cl}^{-}$ions to this molar electrical conductivity : Experimental data ( [77] diamonds), Calculated curve (dashed blue line). Note that the small dots connecting the symbols (squares, circles, diamonds) are only a guide for the eyes. 